\title{
Opinión de los universitarios sobre los cuidados al final de la vida
}

José Manuel Cucalón-Arenal, Alejandro Marín-Ibáñez, Pedro Cía-Gómez, María Guadalupe Blay-Cortés, y grupo de estudiantes de la asignatura de 'Estilos de vida'
Introducción. El progresivo envejecimiento de la población por el aumento de la esperanza de vida, unido a los avances tecnológicos y científicos, hace que la población demande cuidados específicos al final de la vida, con especial cuidado al respeto a sus voluntades y cuidados finales que lleven a lo que se considera una muerte digna y sin sufrimiento.

Materiales y métodos. A través de este estudio descriptivo mediante encuesta conocemos la información que manejan los universitarios y cuáles son sus deseos y opiniones con respecto a la enfermedad terminal y los cuidados al final de la vida, el testamento vital y la declaración de últimas voluntades. Se han encuestado 300 alumnos del Campus Universitario de Zaragoza, estudiantes de facultades de sociales y ciencias.

Resultados. Los universitarios, mayoritariamente mujeres, de religión cristiana y con una edad media de 22 años, desearían ser informadas de su enfermedad por su médico, que tuviera disposición para hablar de su enfermedad y de sus sentimientos. Valoran en su médico especialmente su implicación y comprensión. Desean hablar de su etapa final, de sus deseos, de la donación de órganos y de los cuidados paliativos necesarios aplicados en su domicilio. En caso de fallecimiento preferirían la incineración.

Conclusión. Los jóvenes universitarios no están suficientemente informados sobre los documentos de últimas voluntades (voluntades anticipadas o testamento vital), tienen interés por este tema y esperan de sus médicos y familiares implicación y comprensión ante la enfermedad y la muerte. Desean ser escuchados y tienen opinión sobre lo que se debería hacer cuando llegase el final de su vida.

Palabras clave. Cuidados al final de la vida. Encuesta. Testamento vital. Últimas voluntades. Universitarios.

\section{University students" opinions regarding end-of-life care}

Introduction. The progressive aging of the population, increased life expectancy, coupled with technological and scientific advances mean that the population demands specific care at the end of life, with special care to respect their wishes and care leading to end what has been called a dignified death without suffering.

Materials and methods. Through this study descriptive survey know the information handled by university and what their wishes and opinions regarding terminal illness and care at the end of life, living will and declaration of wills (advance directives). Respondents were 300 students of the University of Zaragoza Campus, students and faculties of social and sciences.

Results. University students, mostly women, of Christian religion with a mean age of 22 years, would like to be informed of your condition by your doctor, you have available to discuss their illness and their feelings. Your doctor especially valued their involvement and understanding. They want to talk about the final stage, their desires, of organ donation. Wish, also, applied necessary palliative care at home. In case of death wish to be cremated.

Conclusion. The university students are not sufficiently informed about the documents of wills (advance directives or living will), have an interest in this issue and expect from their doctors and family involvement and understanding of the disease and death. They want to be heard and have an opinion on what should be done when it came to the end of their lives.
Cátedra SEMG de Estilos de Vida y Promoción de la Salud. Facultad de Medicina. Universidad de Zaragoza. Zaragoza, España.

Correspondencia:

Dr. José Manuel Cucalón Arenal. Cátedra SEMG-Unizar de Estilos de Vida. Facultad de Medicina. Universidad de Zaragoza. Miguel Servet, 51. E-50013 Zaragoza.

E-mail:

jcucalona@semg.es

Grupo de estudiantes de la asignatura de 'Estilos de vida' P. Barrio, A.Y. Brito, A. Coduras, S. Franco, M. García, M. Gómez, C. Lage, M.N. Lozano, T. Martínez, M. Pastor, A.E. Pérez, V. Roda, A.L. Santamaría, M. Torres, R. Valiente.

Agradecimientos: A los estudiantes de nuestra asignatura optativa sobre estilos de vida y promoción de la salud de la Facultad de Medicina de la Universidad de Zaragoza.

Conflicto de intereses: No declarado.

Competing interests: None declared.

(c) 2013 FEM

Key words. Care at the end of the life. College students. Complete wills. Survey. Vital testament. 


\section{Introducción}

El progresivo envejecimiento de la población [1] y el aumento del número de personas con cáncer $\mathrm{u}$ otras enfermedades crónicas o degenerativas [2] representan un reto importante para las sociedades desarrolladas. Muchos de estos enfermos precisarán, al final de su vida, una atención sanitaria esmerada que ayude a afrontar ese final con dignidad y el menor sufrimiento posible [3].

En un mundo altamente tecnificado y con avances científicos muy especializados, sobre todo en medicina, se hace necesario adecuar el derecho de los pacientes a una muerte digna con los cuidados al final de la vida y la aceptación de la voluntad del ciudadano sobre su propia salud, enfermedad y proceso de la muerte $[4,5]$. Las instituciones políticas, en base a una respuesta social, legislan sobre estas materias de interés [6].

Los médicos, especialmente los médicos de familia, deberíamos estar capacitados para ofrecer los cuidados necesarios en esta etapa final de la vida y conocer las herramientas que existen para respetar los derechos de nuestros pacientes. Los estudios realizados indican que existen grandes oportunidades de mejora en cuanto a la información de los profesionales [7-10].

Se debe mantener un profundo respeto a los deseos y voluntades de los pacientes y salvaguardar su intimidad y seguridad ante un proceso que puede estar altamente tecnificado e instrumentalizado con el objetivo de evitar en lo posible el sufrimiento de la persona [11-13].

Algunos estudios han revelado el profundo desconocimiento que la población tiene sobre el testamento vital y el documento de voluntades anticipadas [14-16], lo que indicaría la necesidad de informar y formar a la población con el fin de asegurar el derecho a la libre elección sobre los cuidados al final de la vida que la ley propugna [5]. No hemos encontrado en la revisión realizada estudios descriptivos en base a población joven o universitaria.

Con el estudio hemos pretendido recabar información sobre lo que conocen y piensan los universitarios del Campus de Zaragoza (colectivo juvenil debidamente formado e informado), tanto estudiantes de estudios sociales como de ciencias, sobre la muerte digna, el esfuerzo terapéutico, el testamento vital y el documento de voluntades anticipadas.

\section{Materiales y métodos}

Estudio observacional descriptivo y transversal por medio de encuestas. Se pasó una encuesta a 306 estudiantes del Campus Universitario de Zaragoza que cursaban estudios en tres facultades de ciencias (Medicina, Enfermería y Química) y tres facultades de ciencias sociales (Magisterio, Derecho y Filosofía), por parte de 17 estudiantes que cursaban la asignatura de 'Estilos de vida y comunicación en salud' de la Facultad de Medicina, como trabajo de campo de su asignatura. Las facultades designadas se eligieron por encontrase en el mismo espacio del Campus Universitario y su fácil acceso para los encuestadores, y se desestimaron aquellas otras que se encontraban fuera del campus. Cada alumno de la asignatura seleccionó a 18 alumnos de las facultades referenciadas de forma oportunista, incluyendo estudiantes de cada una de ellas. Se seleccionaron 299 alumnos con encuesta válida. La encuesta constaba de 28 preguntas en varios apartados. Todas eran preguntas cerradas de base dicotómica para facilitar su cumplimentación.

Para el estudio de los datos se construyó una tabla en Excel que contenía todas las encuestas. El análisis se efectuó mediante el programa estadístico SPSS y se realizó un análisis multivariante para valorar la existencia de diferencias entre colectivos por religión, sexo y facultad del encuestado.

\section{Resultados}

La encuesta contenía cinco bloques de preguntas:

\section{Bloque 1. Datos demográficos}

Se seleccionaron 299 estudiantes, 188 mujeres y 111 varones. La edad media era de 22 años (rango: 1843 años). La distribución de estudiantes entre las diferentes facultades fue la siguiente: Medicina, 63 (21,1\%); Enfermería, 45 (15,1\%); Física-Química, 45 (15,1\%); Filosofía, 44 (14,5\%); Derecho, 54 (18,1\%), y Magisterio, 48 (16,1\%).

Respecto a la religión profesada por los encuestados: cristianos, 171 (57,2\%); agnósticos, 109 (36,5\%); otras religiones, 18 (6\%), y musulmanes, $1(0,3 \%)$.

\section{Bloque 2. Información general}

- Conocimiento del testamento vital o documento de voluntades anticipadas: sí, 232 (77,6\%); no, 67 (22,4\%).

- Conocimiento del Registro General de Voluntades Anticipadas de Aragón: sí, 80 (26,8\%); no, 219 (73,2\%).

- Información sobre la diferencia existente entre la 
limitación del esfuerzo terapéutico y la eutanasia activa: sí, 131 (43,8\%); no, 167 (55,9\%).

- ¿Debe existir una legislación específica sobre los cuidados al final de la vida?: sí, 257 (86\%); no, 40 $(13,4 \%)$.

- ¿Sabes dónde y a quién acceder para hablar del testamento vital?: sí, 75 (25,1\%); no, 222 (74,2\%).

Bloque 3. Tus deseos con

relación al final de la vida

- ¿Desearías obtener información completa de tu enfermedad?: sí, 277 (92,6\%); no, 21 (7\%).

- ¿Desearías que tu pareja o familiares próximos fuesen informados de tu enfermedad?: sí, 225 (85,3\%); no, 41 (13,7\%).

- ¿Quién (profesional médico) querrías que te transmitiese esta información?: médico hospitalario, 87 (29,1\%); médico de cabecera, 39 (13\%); ambos facultativos indistintamente, 173 (57,9\%).

- ¿Te gustaría cumplimentar el documento de voluntades anticipadas llegado el momento?: sí, 248 (82,9\%); no, 47 (15,7\%).

- ¿Qué aspecto valorarías más de tu médico de cabecera en la situación de enfermedad terminal?: implicación, 117 (39,1\%); información, 66 (22,1\%); comprensión, 54 (18,1\%); confidencialidad, 37 (12,4\%); dedicación de tiempo, 22 (7,44\%).

- ¿Eres partidario de recibir cuidados paliativos?: sí, 260 (87\%); no, 33 (11\%).

- ¿Qué lugar prefieres para aplicar los cuidados paliativos?: en el domicilio, 195 (65,2\%); en el hospital, 104 (34,8\%).

- ¿Eres partidario de aplicar medidas de limitación del esfuerzo terapéutico?: sí, 234 (78,3\%); no, 59 (19,7\%).

Bloque 4. Información y comunicación de su médico y familiares ante la enfermedad terminal

- ¿Te gustaría que tu médico de cabecera te dedicase tiempo para comentar cuestiones como el documento de voluntades anticipadas, la donación de órganos o la donación del cuerpo a la ciencia?: sí, 261 (87,3\%); otra persona ajena a tu médico, 30 (10\%).

- ¿Te gustaría hablar con tu médico de los sintomas de su enfermedad, su control y evolución, así como de sus cuidados al final de la vida?: sí, 274 (91,6\%); otra persona ajena a tu médico, 20 (6,7\%).

- ¿Consideras positiva o negativa la 'conspiración del silencio, que supone informar a la familia pero no al paciente?: negativa, 220 (73,6\%); positiva, 45 (15,1\%); indiferente, 33 (11\%).
- ¿Crees que es el médico quien debe medir la información a facilitar?: sí, 70 (23,4\%); no, 226 (75,6\%).

- En caso de recibir una mala noticia de tu médico, ¿preferirías que tu pareja o familiar próximo estuviese presente?: estar acompañado, 206 (68,9\%); estar solo, 93 (31,1\%).

- ¿Crees que tu círculo familiar está preparado para hablar contigo de tu situación clínica, deseos $y$ voluntades?: sí, 193 (64,5\%); no, 106 (35,5\%).

- ¿Crees que el médico está adecuadamente preparado para ofrecerte este tipo de información (enfermedad terminal)?: sí, 160 (53,5\%); no, 139 (46,5\%).

- ¿Has compartido información con algún familiar que estuviera en situación de enfermo terminal?: sí, 42 (14\%); no, 204 (68,2\%); no se atreve, 45 (15,1\%).

- ¿Crees que es muy importante que tus deseos y voluntades sean respetados con el máximo rigor tanto por tus familiares como por los médicos que te atienden?: sí, 292 (97,7\%); no, 7 (2,3\%).

\section{Bloque 5. Opinión sobre donación y sepelio}

- ¿Qué desearías que se hiciera con tu cuerpo al fallecer?: incineración, 162 (54,2\%); inhumación, 84 (28,1\%); donación a la ciencia, $51(17,1 \%)$.

- ¿Serías donante de órganos a tu fallecimiento?: sí, 273 (91,3\%); no, 25 (8,4\%).

En el análisis estadístico se investigó si la adscripción a cada una de las facultades a las que pertenecían los alumnos encuestados presentaba o no diferencias significativas que explicasen respuestas divergentes. Tras el oportuno análisis discriminante no se observaron diferencias estadísticamente significativas. Tampoco existieron diferencias en cuanto al sexo ni en cuanto a la religión de los alumnos encuestados.

\section{Discusión}

El avance de la medicina moderna en los cuidados al final de la vida hizo surgir por primera vez el debate sobre las voluntades del paciente, en contraposición a las posibilidades de prolongar la vida de forma fútil, en los años sesenta en Estados Unidos. La primera propuesta realizada por Kutner, un abogado de Chicago, de un documento que regulase los cuidados del final de la vida -living will (testamento vital)- se publicó en 1969 [17].

Tras la oportuna búsqueda bibliográfica sólo hemos podido encontrar un artículo referido a población joven o universitaria. Se trata de un trabajo de fin de máster realizado en estudiantes de ciencias 
de la salud de la Universidad de Granada [18], por lo que es difícil poder extrapolar datos o cruzar resultados. En cambio, sí se encuentran artículos sobre opinión de profesionales, pacientes crónicos o mayores, problemas bioéticos (dilemas éticos) de la limitación del esfuerzo terapéutico y el proceso de la muerte, conocimiento e información ofertada a los pacientes, y técnicas y habilidades en la comunicación con el enfermo terminal.

En 1994 se publicó un estudio multicéntrico norteamericano realizado con 47 pacientes que evaluaba la comunicación entre médicos de atención primaria y sus pacientes sobre los cuidados del final de la vida, que fue desolador [19]. Lo mismo ocurre con otros estudios relacionados con los profesionales, en donde se detectan carencias en la preparación para informar a los pacientes y se indica la necesidad de ofrecer formación e información sobre esta materia [7-12]. En nuestro trabajo, los jóvenes universitarios conceden gran importancia al papel de los sanitarios y aunque la muestra escogida contempla mayoritariamente estudiantes de facultades que deberían conocer sobre este tema (Medicina, Enfermería, Filosofía, Derecho), aún existe un 20$30 \%$ de desconocimiento del documento de voluntades anticipadas y todavía mayor del Registro General de Voluntades Anticipadas y de su tramitación (75\%).

En cuanto a estudios realizados sobre pacientes, destaca el realizado en España en 2005 por nefrólogos a 135 pacientes en tratamiento crónico mediante hemodiálisis, que investigó la opinión en determinadas situaciones relacionadas con el final de la vida de los pacientes. Entre los resultados destaca que un $50 \%$ de los pacientes tratados mediante hemodiálisis periódica son partidarios de limitar ciertos tratamientos en circunstancias de pronóstico infausto, siendo los enfermos más ancianos los más partidarios a manifestar la voluntad sobre esas limitaciones [20]. En nuestro estudio, ocho de cada diez jóvenes universitarios son partidarios de recibir cuidados paliativos y de aplicar la limitación al esfuerzo terapéutico.

En otro estudio realizado sobre pacientes mayores de 18 años (edad media: 54 años) entrevistados en un centro de salud de Valencia, sólo el $4 \%$ de ellos tenían hecho el documento de voluntades anticipadas, cuya existencia desconocían el 66\% de los entrevistados. Tras la explicación del documento por el médico de familia, el 96\% lo entendían correctamente [17]. En otro estudio realizado en el año 2010 en un centro de salud de Murcia, el 75\% de los encuestados desconocían el documento de voluntades anticipadas, aunque un $90 \%$ creían que era un documento útil [21]. Por último, en el estudio de López-Rey et al en pacientes que acudían a un centro de salud en Toledo (edad media: 46 años), el $88 \%$ desconocían el documento de voluntades anticipadas -sin diferencia de sexo ni creencias religiosas-, casi un 50\% donarían sus órganos y el $35 \%$ donarían los de algún familiar [15]. Lo mismo sucede en otros trabajos realizados en otras ciudades. Estos datos se acentúan en los jóvenes de nuestro estudio, que son mayoritariamente donantes futuros de órganos, lo que podría ofrecer alguna explicación de que nuestro país esté a la cabeza del mundo en esta práctica. Al igual que en el estudio en centros de salud, tampoco hemos constatado diferencias por religión ni sexo. No obstante, es llamativo encontrar en nuestro trabajo un 36\% de agnósticos, lo que puede contrastar con la religión profesada por nuestros progenitores, mayoritariamente cristiana.

En estudios realizados en personas mayores (en residencias y centros de salud) destacan como principales conclusiones que los ancianos prefieren la planificación anticipada de decisiones al testamento vital. Su opinión sobre el testamento vital se asocia a la vivencia singular de otros eventos en el final de la vida. Conceden un papel relevante a la familia y desean conducir su propia vida, pero 'sin papeles escritos' [22].

Por último, en población general, destaca el trabajo de Andalucía sobre un total de 2.300 encuestados (obtenidos del barómetro sanitario en Andalucía 2007), en el que se estima que el $80 \%$ de los encuestados desconocen el documento de voluntades anticipadas o el testamento vital, y de los informados, la mayor parte lo fueron por los medios de comunicación (sólo un 10\% a través del personal sanitario). Lo valoran de forma positiva la mayor parte de la población y se establecen diferencias en cuanto a la edad, siendo los más jóvenes (18-30 años) los que más conocen el documento de voluntades anticipadas, los más favorables al tratamiento paliativo y a la limitación del esfuerzo terapéutico para no alargar la vida de forma innecesaria, y los más favorables a la eutanasia en casos especiales (enfermedad terminal...). No se detectan diferencias por religión profesada.

En el estudio de Granada [18], realizado sobre estudiantes de enfermería, fisioterapia y terapia ocupacional (ciencias de la salud) de la Universidad de Granada, se aprecia que la mitad de los encuestados conocían el documento de voluntades anticipadas y la ley que lo regula en Andalucía, pero ignoraban la aplicación de la limitación al esfuerzo terapéutico. El $83 \%$ no se consideraban capacitados 
para informar sobre el testamento vital o el documento de voluntades anticipadas y más del $60 \%$ no se hallaban capacitados para tratar con el enfermo terminal. Estos datos se asemejan a los nuestros en cuanto a conocimiento del testamento vital (en el nuestro llegan al 77\%) y ponen de relieve la falta de preparación de los profesionales en este sentido, así como que son necesarios curso formativos tanto para la población general como para los profesionales implicados.

Por tanto, nuestro trabajo se desarrolla en población poco encuestada o encuestada subliminalmente y de ahí el interés que puede suscitar conocer la opinión de los universitarios, subgrupo de población joven con una, supuestamente, mejor preparación formativa.

La falta de información sobre el documento de voluntades anticipadas de nuestro estudio es común al resto de grupos poblacionales, pero se aprecian en los jóvenes algunas peculiaridades: dan mucha importancia a temas como la calidad de vida, el esfuerzo terapéutico, la información de familiares y la comunicación con los profesionales sobre aspectos relacionados con el final de la vida. Este trabajo abre canales de información que deben ampliarse en posteriores estudios más específicos, pero ofrece información oportuna e interesante sobre cuestiones antes no tratadas. Ofrece al mismo tiempo datos de gran interés para la salud pública futura relacionados con la planificación sanitaria (donación de órganos y tratamiento de los cuerpos tras el fallecimiento).

En conclusión, en cuanto a los resultados destacan dos cuestiones. En primer lugar, la información y el conocimiento de la población joven sobre los documentos de últimas voluntades son escasos y sería interesante trabajar sobre este aspecto. En segundo lugar, los universitarios dan mucha importancia a la información de su proceso, la correcta comunicación con profesionales y familiares (miedos, reticencias, valores...), defienden la aplicación de unos correctos cuidados paliativos y que éstos se procuren en su domicilio mientras pueda ser factible. Se debe incidir en la diferencia entre limitación del esfuerzo terapéutico (eutanasia pasiva) y eutanasia activa. Por consiguiente, los jóvenes demandan información y demuestran estar interesados en las cuestiones relacionadas con el final de la vida. Creemos necesario ampliar información mediante estudios cualitativos sobre la opinión de los jóvenes universitarios en estos temas relacionados con el proceso de morir, que permitan ofrecer formación en los respectivos currículos formativos en las diferentes facultades españolas. Destaca un preocupante desconocimiento del documento de voluntades anticipadas y del testamento vital que debe corregirse. Nuestro trabajo abre nuevos interrogantes que deberán abordarse de una manera más específica.

\section{Bibliografía}

1. Pérez-Díaz J. Las causas del envejecimiento demográfico. Apuntes de Demografía. URL: http://apuntesdedemografia. wordpress.com/envejecimiento-demografico/que-es/ las-causas-del-envejecimiento-demografico. [31.01.2013].

2. Serrano-González MI. La educación para la salud del siglo XXI: comunicación y salud. 2 ed. Madrid: Díaz de Santos; 2002.

3. González-Barón M. Tratado de medicina paliativa y tratamiento de soporte del paciente con cáncer. Madrid: Panamericana; 2007.

4. Barrio-Cantalejo IM, Simón-Lorda P, Júdez-Gutiérrez J. De las voluntades anticipadas o instrucciones previas a la planificación anticipada de las decisiones. Nure Investigación 2004; 5 .

5. Extracto de la legislación española, artículo 11.1 de la Ley 41/2002, de 14 de noviembre, básica reguladora de la autonomía del paciente y de derechos y obligaciones en materia de información y documentación clínica. Boletín Oficial del Estado, 15 de noviembre de 2002.

6. Lauzirika N. Los médicos de atención primaria, eslabón clave en los testamentos vitales. El Médico, 16 de noviembre de 2007. p. 14-20 y 49.

7. Angora-Mazuelos F. Voluntades anticipadas vs. instrucciones previas o testamento vital en atención primaria de salud. Rev Clin Med Fam 2008; 2: 210-5.

8. Santos C, Forn MA, Pérez R, Corrales A, Urgarriza L, Sales C. ¿Estamos preparados los médicos de familia para ayudar a nuestros pacientes a hacer el testamento vital? Revista de Calidad Asistencial 2007; 22: 262-5.

9. Valle-Sánchez A, Farráis-Villalba S, González-Romero PM, Galindo-Barragán S, Rufino-Delgado MT, Marco-García MT. Documento de voluntades anticipadas: opinión de los profesionales sanitarios de atención primaria. SEMERGEN 2009; 35: 111-4.

10. Valencia-Montoya G. ¿Salvar la vida o prolongar la agonía? Cultura del Cuidado de Enfermería 2010; 7: 1.

11. Mateos-Rodríguez A, Huerta-Arroyo A, Benito-Bellisca MA. Instrucciones previas. Actitud de los profesionales de emergencias. Revista de la Sociedad Española de Medicina de Urgencias y Emergencias 2007; 19: 241-4.

12. Yagüe-Sánchez JM. Las enfermeras ante las voluntades anticipadas: un reto jurídico, ético y práctico. Rev Enfermería 2012; 6: 43-50.

13. Marcos del Cano AM. Las voluntades anticipadas. In Elizari FJ, coord. 10 palabras claves al final de la vida. Estella: Verbo Divino; 2007. p. 389-425.

14. Martínez-Ortega JC, Martínez-Ortega RM, Oter-Quintana C, Rubiales-Paredes D. Testamento vital: la toma de decisiones en el final de la vida. Revista Rol de Enfermería 2007; 30: 192-9.

15. Ángel López-Rey E, Romero-Cano M, Tébar-Morales JP, Mora-García C, Fernández-Rodríguez O. Conocimiento y actitudes de la población ante el documento de voluntades anticipadas. Enferm Clin 2008; 18: 115-9.

16. Serrano del Rosal R., Ranchal-Romero J, García de Diego JM, Galiano-Coronil S, Biedma-Velázquez L. Documento de trabajo: opiniones y actitudes de la población en Andalucía sobre testamento vital, muerte digna y eutanasia. Córdoba: Instituto de Estudios Sociales Avanzados; 2007.

17. Ruiz-Carbonell MA. Análisis del conocimiento, comprensión y valoración que los pacientes de un centro de salud tienen sobre el documento de voluntades anticipadas (DVA). Barcelona: Universitat Autònoma de Barcelona; 2010.

18. Morales-Martín AM, Schmidt-Riovalle J, García-García I. Conocimiento del alumnado de ciencias de la salud sobre la 
ley andaluza de muerte digna. Investigación y Educación en Enfermería 2012; 30: 2.

19. Pfeifer MP, Sidorov JE, Smith AC, Boero JF, Evans AT, Settle MB. The discussion of end of life medical care by primary care patients and physicians: a multicenter study using structured qualitative interviews. The EOL Study Group. J Gen Intern Med 1994; 9: 82-8.

20. Rodríguez-Jornet A, Ibeas J, Real J, Pena S, Martínez-Ocaña JC, García-García M. Documento de voluntades anticipadas de pacientes con insuficiencia renal crónica terminal en tratamiento sustitutivo mediante diálisis. Nefrología 2007; 27: 581-92.

21. Serrano-Teruel R, López-López R, Cardenal-González I, Illana-Rodríguez J. Conocimiento e información sobre el documento de instrucciones previas en un centro de salud urbano de Murcia. Aten Primaria 2011; 43: 680-1.

22. Barrio-Cantalejo I, Barreiro-Bello JM, Pascau GonzálezGarzón MJ, Simón-Lorda P, Güémez-Abad MP, RIMARED G03/100. La perspectiva de las personas mayores sobre el testamento vital. Index Enferm 2006; 16: 52-3. 